Thermodynamics and spin-charge separation of one-dimensional strongly repulsive threecomponent fermions

This article has been downloaded from IOPscience. Please scroll down to see the full text article.

2011 J. Phys. A: Math. Theor. 44405005

(http://iopscience.iop.org/1751-8121/44/40/405005)

View the table of contents for this issue, or go to the journal homepage for more

Download details:

IP Address: 150.203.179.232

The article was downloaded on 04/10/2011 at 01:02

Please note that terms and conditions apply. 


\title{
Thermodynamics and spin-charge separation of one-dimensional strongly repulsive three-component fermions
}

\author{
Peng He $\mathbf{H e}^{1,2}$, Jen Yee Lee ${ }^{2}$, Xiwen Guan ${ }^{2}$, Murray T Batchelor ${ }^{2,3}$ \\ and Yupeng Wang ${ }^{1}$ \\ ${ }^{1}$ Beijing National Laboratory for Condensed Matter Physics, Institute of Physics, \\ Chinese Academy of Sciences, Beijing 100190, People's Republic of China \\ 2 Department of Theoretical Physics, Research School of Physics and Engineering, \\ Australian National University, Canberra ACT 0200, Australia \\ ${ }^{3}$ Mathematical Sciences Institute, Australian National University, Canberra ACT 0200, Australia \\ E-mail: hepeng@iphy.ac.cn
}

Received 10 May 2011

Published 15 September 2011

Online at stacks.iop.org/JPhysA/44/405005

\begin{abstract}
The low-temperature thermodynamics of one-dimensional strongly repulsive $S U$ (3) fermions in the presence of a magnetic field is investigated via the Yang-Yang thermodynamic Bethe ansatz method. The analytical free energy and magnetic properties of the model at low temperatures in a weak magnetic field are derived via the Wiener-Hopf method. It is shown that the low-energy physics can be described by spin-charge separated conformal field theories of an effective Tomonaga-Luttinger liquid and an antiferromagnetic $S U$ (3) Heisenberg spin chain. Beyond the Tomonaga-Luttinger liquid regime, the equation of state is given in terms of the polylog function for a weak external field. The results obtained are essential for further study of quantum criticality in strongly repulsive three-component fermions.
\end{abstract}

PACS numbers: 03.75.Ss, 03.75.Hh, 02.30.IK, 05.30.Fk

\section{Introduction}

Recent experiments on quantum gases in one dimension (1D) [1-5] provide an exciting way to test 1D many-body physics extensively studied in the literature via the Bethe ansatz (BA) [6], effective field theory [7] and other theoretical methods. Novel 1D many-body physics such as the phenomena of spin-charge separation [8-10], universal Luttinger liquid thermodynamics [11-15] and quantum criticality are quite different from higher dimensional physics. 1D manybody systems also provide insights into higher dimensional physics. For example, theoretical predictions for the existence of a Fulde-Ferrell-Larkin-Ovchinnikov (FFLO) [16] like pairing state in the 1D interacting Fermi gas have emerged [13, 17-27]. 
A scheme for mapping out the physical properties of homogeneous systems by the inhomogeneity of the trap has been successfully applied to ultracold atom experiments [5], since the thermodynamics of interacting fermionic systems can be measured explicitly and the interaction between fermions can be tuned precisely via a broad Feshbach resonance. In particular, fermionic alkaline-earth atoms display an exact $S U(N)$ spin symmetry with $N=2 I+1$ where $I$ is the nuclear spin [28]. For example, a recent experiment dramatically realized the model of fermionic atoms with $S U(2) \times S U(6)$ symmetry where electron spin decouples from its nuclear spin $I=5 / 2$ for ${ }^{171} \mathrm{Yb}$ [29]. Such fermionic systems with enlarged $S U(N)$ spin symmetry are expected to display a remarkable diversity of new quantum phases and quantum critical phenomena due to the existence of multiple charge bound states $[30,31]$. The strongly attractive multi-component Fermi gases with higher spin symmetries have also been investigated via the BA method [32-34]. These developments provide an exciting opportunity to explore the universal thermodynamics and quantum critical behavior of strongly interacting fermions with high spin symmetries in 1D.

The BA has proven to be a powerful method to study 1D quantum gases. This method was applied to the 1D Bose gas by Lieb and Liniger [35] and to the spin-1/2 Fermi gas by Yang [36] and Gaudin [37]. After that, the multi-component Fermi gas was studied by Sutherland [38]. The study of the thermodynamics of the attractive Fermi gas was initiated by Yang [39] and Takahashi [40]. Moreover, the thermodynamics of 1D Fermi gases is also widely studied for its rich physics. For spin- $1 / 2$ fermions with attractive interaction, the phase diagrams and Luttinger liquid physics have been investigated using both analytical $[13,14]$ and numerical methods $[18,17]$. The key features of this $T=0$ phase diagram were experimentally confirmed using finite temperature density profiles of trapped fermionic ${ }^{6} \mathrm{Li}$ atoms [5].

For the strongly attractive spin-1/2 Fermi gas at finite temperatures, the thermodynamics of the homogeneous system is described by two coupled Fermi gases of bound pairs and excess fermions in the charge sector and ferromagnetic spin-spin interaction in the spin sector $[13,14]$. Spin fluctuations are suppressed by a strong effective magnetic field at low temperatures. However, for the repulsive case, the situation is quite different since spin fluctuations play a dominant role in the low-energy physics. Spin fluctuations are described by the $S U$ (2) antiferromagnetic spin-spin Heisenberg system and thus the coupling of charge and spin parts make the thermodynamic Bethe ansatz (TBA) equations more complex. So far we know that the Wiener-Hopf method can be applied here to deal with the spin part of the TBA equations for weak magnetic field. This method was used by Mezincescu $e t$ al to investigate the thermodynamics of Heisenberg spin chains with $S U(2)$ and $S U(3)$ symmetries [41, 42]. Recently, Lee et al [15] applied the Wiener-Hopf method to spin-1/2 repulsive fermions with $S U$ (2) symmetry and studied the low-temperature thermodynamics and correlation functions via the BA method analytically. The Wiener-Hopf technique was applied to obtain the solutions of the TBA equations in the spin sector. Then Sommerfeld's expansion was utilized in the lowtemperature limit with strong interactions to get the multi-component Tomonaga-Luttinger liquid (TLL) form of the free energy, which shows a spin-charge separation with the central charge of the spin and charge parts both equal to 1 .

Spin-charge separation is a hallmark of 1D many-body physics. It is a universal feature that interacting particles 'split' into spins and charges as temperature tends to absolute zero temperature. The collective excitations with only spin or charge are called spinon and chargon/holon (the antiparticle of chargon), which have different velocities. This behavior was studied in different kinds of materials [8-10] and recently observed in experiments, for example, in 1D metallic wires on surfaces, 1D organic wires, carbon-nanotubes, quantum wires in semiconductors, and other types of 1D systems. 1D quantum systems with high 
spin symmetries will give diverse magnetism and exotic properties for these kinds of gases. However, such systems are more difficult to solve $[43,44,45]$ due to the complicated magnetic ordering. The three-component Fermi gas has $U(1) \times S U(3)$ symmetry which leads to two sets of spin waves. It is very interesting to see how the low-temperature thermodynamics of such a gas naturally separates into free Gaussian field theories for the $U(1)$ charge degree of freedom and the two spin sectors.

In this paper, 1D fermions with $U(1) \times S U(3)$ symmetry are studied using the WienerHopf method. The result shows that the system with strong repulsive interactions at low temperatures has the central charge $C_{c}=1$ for the charge sector and central charge $C_{s}=2$ for the two spin sectors. We also derive the leading-order finite-temperature corrections of the free energy which is consistent with expectations from conformal field theory [11, 12, 46, 49]. In addition, the polylog function is used to derive the equation of state in a wider temperature regime.

This paper is organized as follows. In section 2, the BA and TBA equations are presented. In section 3, the Wiener-Hopf method is applied to solve the TBA equations for the spin sectors. In section 4, the thermodynamics of the system is derived via Sommerfeld's expansion for low temperatures and strong interactions. We also show that the expression for the free energy at low temperatures is in agreement with conformal field theory and derive the spin and charge velocities. Section 5 is reserved for the conclusion and discussion. Some details are given in the appendices.

\section{The model and thermodynamic BA}

Consider a 1D system of $N$ fermions with mass $m$ and a spin independent $\delta$-function potential. The fermions can occupy three possible hyperfine levels $(|1\rangle,|2\rangle$ and $|3\rangle)$ with particle numbers $N^{1}, N^{2}$ and $N^{3}$, respectively. They are also constrained to a line of length $L$ with periodic boundary conditions. This system is described by the many-body Hamiltonian $[38,40]$

$$
\mathcal{H}_{0}=-\frac{\hbar^{2}}{2 m} \sum_{i=1}^{N} \frac{\partial^{2}}{\partial x_{i}^{2}}+g_{1 D} \sum_{1 \leqslant i<j \leqslant N} \delta\left(x_{i}-x_{j}\right)+E_{z} .
$$

Here $E_{z}=\sum_{i=1}^{3} N^{i} \epsilon_{Z}^{i}$ is the Zeeman energy. The spin-independent contact interaction $g_{1 D}$ exists between fermions with different hyperfine states so that the number of fermions in each spin state is conserved. It is positive for repulsive interaction and negative for attractive interaction. For simplicity, we set $\hbar=2 m=1$. They can be reintroduced when necessary. It is possible to tune scattering lengths between atoms in different sublevels to form nearly $S U$ (3) degenerate Fermi gases via a broad Feshbach resonance.

The Hamiltonian in equation (1) exhibits a symmetry of $U(1) \times S U(3)$, where $U(1)$ and $S U$ (3) are the symmetries for charge and spin degrees of freedom, respectively. As mentioned earlier, this model was solved via a nested BA $[38,40]$. The energy eigenspectrum is given by $E=\frac{\hbar^{2}}{2 m} \sum_{j=1}^{N} k_{j}^{2}$ in terms of the quasimomenta $\left\{k_{j}\right\}$ satisfying the BA equations $[38,40]$

$$
\begin{aligned}
& \mathrm{e}^{\mathrm{i} k_{j} L}=\prod_{\ell=1}^{M_{1}} \frac{k_{j}-\Lambda_{\ell}+\mathrm{i} c / 2}{k_{j}-\Lambda_{\ell}-\mathrm{i} c / 2}, \\
& \prod_{j=1}^{N} \frac{\Lambda_{\ell}-k_{j}+\mathrm{i} c / 2}{\Lambda_{\ell}-k_{j}-\mathrm{i} c / 2}=-\prod_{\alpha=1}^{M_{1}} \frac{\Lambda_{\ell}-\Lambda_{\alpha}+\mathrm{i} c}{\Lambda_{\ell}-\Lambda_{\alpha}-\mathrm{i} c} \prod_{m=1}^{M_{2}} \frac{\Lambda_{\ell}-\lambda_{m}-\mathrm{i} c / 2}{\Lambda_{\ell}-\lambda_{m}+\mathrm{i} c / 2}, \\
& \prod_{\ell=1}^{M_{1}} \frac{\lambda_{m}-\Lambda_{\ell}+\mathrm{i} c / 2}{\lambda_{m}-\Lambda_{\ell}-\mathrm{i} c / 2}=-\prod_{\beta=1}^{M_{2}} \frac{\lambda_{m}-\lambda_{\beta}+\mathrm{i} c}{\lambda_{m}-\lambda_{\beta}-\mathrm{i} c} .
\end{aligned}
$$


Here $j=1, \ldots, N, \ell=1, \ldots, M_{1}$ and $m=1, \ldots, M_{2}$ with quantum numbers $M_{1}=N^{2}+N^{3}$ and $M_{2}=N^{3}$. We define the interaction strength as $c=m g_{1 D} / \hbar^{2}>0$ since we only consider the repulsive case. The parameters $\left\{k_{j}\right\}$ are quasimomenta and $\left\{\Lambda_{\ell}, \lambda_{m}\right\}$ are the rapidities that characterize the internal hyperfine spin degrees of freedom. In the thermodynamic limit, $N, L \rightarrow \infty$ with the ratio for linear particle density $n=N / L$ kept finite. Note that there are two classes of strings. Both sets of solutions can be expressed in terms of the string hypothesis

$$
\begin{array}{ll}
\Lambda_{j}^{n, \alpha}=\Lambda_{j}^{n}-\frac{1}{2}(n+1-2 \alpha) \mathrm{i}|c|, & \alpha=1, \ldots, n \\
\lambda_{j}^{n, \beta}=\lambda_{j}^{n}-\frac{1}{2}(n+1-2 \beta) \mathrm{i}|c|, & \beta=1, \ldots, n,
\end{array}
$$

where $n$ is the length of each string, $j$ labels each individual string, and $\Lambda_{j}^{n}$ and $\lambda_{j}^{n}$ are the real parts of the $\Lambda$ and $\lambda$ strings, respectively. To distinguish the rapidities in different spin levels, define $\lambda^{(r)}$ as

$$
\Lambda:=\lambda^{(1)}, \quad \lambda:=\lambda^{(2)} .
$$

For repulsive interaction, the quasimomenta $\left\{k_{j}\right\}$ are real, while $\left\{\lambda^{(r)}\right\}$ form complex spin strings which characterize the spin wave fluctuations at finite temperatures. Without loss of generality, we consider the case where equal Zeeman splitting occurs between fermions of different spin to simplify our analysis, i.e. $H_{1}=H_{2}=H$. The equilibrium states are then determined by minimizing the Gibbs free energy, which gives rise to a set of coupled nonlinear integral equations - the TBA equations, which are

$$
\begin{aligned}
& \varepsilon(k)=k^{2}-\mu-H-T \sum_{n=1}^{\infty} a_{n} * \ln \left(1+\mathrm{e}^{-\phi_{n}^{(1)}(k) / T}\right), \\
& \phi_{n}^{(1)}\left(\lambda^{(1)}\right)=n H-T a_{n} * \ln \left(1+\mathrm{e}^{-\varepsilon\left(\lambda^{(1)}\right) / T}\right)+T \sum_{m} T_{m n} * \ln \left(1+\mathrm{e}^{-\phi_{n}^{(1)}\left(\lambda^{(1)}\right) / T}\right) \\
& -T \sum_{m} S_{m n} * \ln \left(1+\mathrm{e}^{-\phi_{n}^{(2)}\left(\lambda^{(1)}\right) / T}\right), \\
& \phi_{n}^{(2)}\left(\lambda^{(2)}\right)=n H+T \sum_{m} T_{m n} * \ln \left(1+\mathrm{e}^{-\phi_{n}^{(2)}\left(\lambda^{(2)}\right) / T}\right)-T \sum_{m} S_{m n} * \ln \left(1+\mathrm{e}^{-\phi_{n}^{(1)}\left(\lambda^{(2)}\right) / T}\right) .
\end{aligned}
$$

Here $\varepsilon(k)$ denotes the dressed energy and $\phi_{n}^{(r)}(r=1,2)$ are associated with densities of strings with length- $n$ in $\lambda^{(r)}$ parameter spaces. $*$ represents the convolution integral, i.e. $(a * b)(x)=\int a(x-y) b(y) \mathrm{d} y$, and the function $a_{m}(x)$ is given by

$$
a_{m}(x)=\frac{1}{2 \pi} \frac{m c}{(m c / 2)^{2}+x^{2}} .
$$

The functions $T_{m n}$ and $S_{m n}$ are defined as

$$
\begin{aligned}
& T_{m n}(x)= \begin{cases}a_{m+n}(x)+2 a_{m+n-2}(x)+\cdots+2 a_{|m-n|+2}(x)+a_{|m-n|}(x), & \text { for } m \neq n ; \\
2 a_{2}(x)+2 a_{4}(x)+\cdots+2 a_{2 n-2}(x)+a_{2 n}(x), & \text { for } m=n .\end{cases} \\
& S_{m n}(x)= \begin{cases}a_{m+n-1}(x)+a_{m+n-3}(x)+\cdots+a_{|m-n|+3}(x)+a_{|m-n|+1}(x), & \text { for } m \neq n ; \\
a_{1}(x)+a_{3}(x)+\cdots+a_{2 n-3}(x)+a_{2 n-1}(x), & \text { for } m=n .\end{cases}
\end{aligned}
$$

An alternative set of TBA equations are

$$
\begin{aligned}
& \varepsilon(k)=k^{2}-\mu-T s_{1} * a_{1} * \ln \left(1+\mathrm{e}^{-\varepsilon(k) / T}\right) \\
& -T s_{1} * \ln \left(1+\mathrm{e}^{\phi_{1}^{(1)}(k) / T}\right)-T s_{2} * \ln \left(1+\mathrm{e}^{\phi_{1}^{(2)}(k) / T}\right), \\
& \phi_{1}^{(1)}\left(\lambda^{(1)}\right)=T s * \ln \left(1+\mathrm{e}^{\phi_{2}^{(1)}\left(\lambda^{(1)}\right) / T}\right)-T s * \ln \left(1+\mathrm{e}^{-\varepsilon\left(\lambda^{(1)}\right) / T}\right)-T s * \ln \left(1+\mathrm{e}^{-\phi_{1}^{(2)}\left(\lambda^{(1)}\right) / T}\right), \\
& \phi_{n}^{(1)}\left(\lambda^{(1)}\right)=T s * \ln \left(1+\mathrm{e}^{\phi_{n-1}^{(1)}\left(\lambda^{(1)}\right)}\right)+T s * \ln \left(1+\mathrm{e}^{\phi_{n+1}^{(1)}\left(\lambda^{(1)}\right)}\right), \\
& \phi_{1}^{(2)}\left(\lambda^{(2)}\right)=T s * \ln \left(1+\mathrm{e}^{\phi_{2}^{(2)}\left(\lambda^{(2)}\right) / T}\right)-T s * \ln \left(1+\mathrm{e}^{-\phi_{1}^{(1)}\left(\lambda^{(2)}\right) / T}\right), \\
& \phi_{n}^{(2)}\left(\lambda^{(2)}\right)=T s * \ln \left(1+\mathrm{e}^{\phi_{n-1}^{(2)}\left(\lambda^{(2)}\right)}\right)+T s * \ln \left(1+\mathrm{e}^{\phi_{n+1}^{(2)}\left(\lambda^{(2)}\right)}\right),
\end{aligned}
$$


with the limit

and

$$
\lim _{n \rightarrow \infty} \frac{\phi_{n}^{(r)}\left(\lambda^{(r)}\right)}{n}=H \quad(r=1,2)
$$

$$
\begin{aligned}
& s(x)=\frac{1}{2 c \cosh (\pi x / c)}, \\
& s_{1}(x)=\frac{1}{\sqrt{3} c} \frac{\cosh (\pi x / 3 c)}{\cosh (\pi x / c)}, \\
& s_{2}(x)=\frac{1}{\sqrt{3} c} \frac{\sinh (\pi x / 3 c)}{\sinh (\pi x / c)} .
\end{aligned}
$$

The two sets of TBA equations are interchangeable via Fourier transformation.

\section{Wiener-Hopf solution for the spin part}

The string part is the most difficult part of the TBA equations to solve. It consists of an infinite number of string functions $\phi_{n}^{(r)}(\lambda)$ which are related through the set of equations (6) or (10). These coupled nonlinear integral equations cannot be solved analytically in most cases. However, in some special cases the equations can be simplified. When $T$ is very low $(T \ll 1)$, these equations reduce to a set of linearly coupled equations. Moreover, when $c$ is very large $(c \gg 1)$, Taylor expansion can be applied to solve this set of equations. In the following, we will consider the case where $T \ll 1$ and $c \gg 1$.

Observe from the second set of TBA equations (10) that $\phi_{n}^{(r)}>0$ for $n>1$ at low temperatures. The functions $s(\lambda)$ and $\ln \left(1+\mathrm{e}^{\phi_{n}^{(r)}(\lambda) / T}\right)$ are always greater than zero in their entire domain, so the convolution is always greater than zero. This positivity condition implies that the function $T \ln \left(1+\mathrm{e}^{-\phi_{n}^{(r)}(\lambda) / T}\right) \rightarrow 0$ for $T \rightarrow 0$. Thus, all the spin string functions could be neglected except the $n=1$ function $\phi_{1}^{(r)}(\lambda)$ in the first set of TBA equations. For $T \ll 1$, only the lowest strings are therefore left in the strong coupling limit $c \gg 1$, and we can rewrite the term as

$$
T a_{1} * \ln \left(1+\mathrm{e}^{-\varepsilon(k) / T}\right) \approx 2 \pi P a_{1}(k)
$$

where

$$
P=\frac{T}{2 \pi} \int_{-\infty}^{\infty} \mathrm{d} k \ln \left(1+\mathrm{e}^{-\varepsilon(k) / T}\right) .
$$

The first set of TBA equations (6) can thus be simplified as

$$
\begin{aligned}
& \varepsilon(k)=k^{2}-\mu-H-T a_{1} * \ln \left(1+\mathrm{e}^{-\phi_{1}^{(1)}(k) / T}\right), \\
& \phi_{1}^{(1)}(\lambda)=H-2 \pi P a_{1}(\lambda)+T a_{2} * \ln \left(1+\mathrm{e}^{-\phi_{1}^{(1)}(\lambda) / T}\right)-T a_{1} * \ln \left(1+\mathrm{e}^{-\phi_{1}^{(2)}(\lambda) / T}\right), \\
& \phi_{1}^{(2)}(\lambda)=H+T a_{2} * \ln \left(1+\mathrm{e}^{-\phi_{1}^{(2)}(\lambda) / T}\right)-T a_{1} * \ln \left(1+\mathrm{e}^{-\phi_{1}^{(1)}(\lambda) / T}\right),
\end{aligned}
$$

where we have switched to a common spin variable $\lambda$ to represent both spin spaces $\lambda^{(1)}$ and $\lambda^{(2)}$. Taking the limit $T \rightarrow 0$ yields

$$
\begin{aligned}
& \varepsilon(k)=k^{2}-\mu-H+a_{1} * \phi_{1}^{(1)-}(k), \\
& \phi_{1}^{(1)}(\lambda)=H-2 \pi P a_{1}(k)-a_{2} * \phi_{1}^{(1)-}(\lambda)+a_{1} * \phi_{1}^{(2)-}(\lambda), \\
& \phi_{1}^{(2)}(\lambda)=H-a_{2} * \phi_{1}^{(2)-}(\lambda)+a_{1} * \phi_{1}^{(1)-}(\lambda) .
\end{aligned}
$$


We have decomposed the functions as

$$
\phi_{1}^{(r)}(\lambda)=\phi_{1}^{(r)+}(\lambda)+\phi_{1}^{(r)-}(\lambda)
$$

where

$$
\begin{aligned}
& \phi_{1}^{(r)+}(\lambda)= \begin{cases}\phi_{1}^{(r)}(\lambda), & \text { for } \quad \phi_{1}^{(r)}(\lambda)>0 \\
0, & \text { for } \quad \phi_{1}^{(r)}(\lambda)<0\end{cases} \\
& \phi_{1}^{(r)-}(\lambda)= \begin{cases}0, & \text { for } \phi_{1}^{(r)}(\lambda)>0 \\
\phi_{1}^{(r)}(\lambda), & \text { for } \phi_{1}^{(r)}(\lambda)<0\end{cases}
\end{aligned}
$$

After taking the Fourier transforms and going through some manipulation, equations (21) and (22) can be written as

$$
\begin{aligned}
& \phi_{1}^{(1)}(\lambda)=H-2 \pi P s_{1}(\lambda)+h * \phi_{1}^{(1)+}(\lambda)+g * \phi_{1}^{(2)+}(\lambda), \\
& \phi_{1}^{(2)}(\lambda)=H-2 \pi P s_{2}(\lambda)+h * \phi_{1}^{(2)+}(\lambda)+g * \phi_{1}^{(1)+}(\lambda),
\end{aligned}
$$

where

$$
\begin{aligned}
& h(\lambda)=s_{1} * a_{1}(\lambda)-s_{2}(\lambda), \\
& g(\lambda)=s_{2} * a_{1}(\lambda)-s_{1}(\lambda) .
\end{aligned}
$$

Both equations in (26) are the $T \rightarrow 0$ limit cases of $\phi_{1}^{(1)}(\lambda)=H-2 \pi P s_{1}(\lambda)+h * T \ln \left(1+\mathrm{e}^{\phi_{1}^{(1)}(\lambda) / T}\right)+g * T \ln \left(1+\mathrm{e}^{\phi_{1}^{(2)}(\lambda) / T}\right)$, $\phi_{1}^{(2)}(\lambda)=H-2 \pi P s_{2}(\lambda)+h * T \ln \left(1+\mathrm{e}^{\phi_{1}^{(2)}(\lambda) / T}\right)+g * T \ln \left(1+\mathrm{e}^{\phi_{1}^{(1)}(\lambda) / T}\right)$.

In order to find a relationship between the dressed energies and the temperature, we write $\phi_{1}^{(1)}(\lambda)$ as

$$
\phi_{1}^{(r)}(\lambda)=\phi^{(r)}(\lambda)+\eta^{(r)}(\lambda),
$$

where the first term $\phi^{(r)}(\lambda)$ is the term of zeroth order when $T=0$ and second term $\eta^{(r)}(\lambda)$ is the first-order correction to the limit $T \rightarrow 0$. Substituting this equation into (29) and using the expressions in (26) for $\phi^{(r)}(\lambda)$, the equation for $\eta^{(r)}(\lambda)$ becomes

$$
\begin{aligned}
\eta^{(1)}(\lambda)=h *[ & \left.T \ln \left(1+\mathrm{e}^{\left(\phi^{(1)}(\lambda)+\eta^{(1)}(\lambda)\right) / T}\right)-\phi^{(1)+}(\lambda)\right] \\
+ & g *\left[T \ln \left(1+\mathrm{e}^{\left(\phi^{(2)}(\lambda)+\eta^{(2)}(\lambda)\right) / T}\right)-\phi^{(2)+}(\lambda)\right], \\
\eta^{(2)}(\lambda)=h *[ & \left.T \ln \left(1+\mathrm{e}^{\left(\phi^{(2)}(\lambda)+\eta^{(2)}(\lambda)\right) / T}\right)-\phi^{(2)+}(\lambda)\right] \\
+ & g *\left[T \ln \left(1+\mathrm{e}^{\left(\phi^{(1)}(\lambda)+\eta^{(1)}(\lambda)\right) / T}\right)-\phi^{(1)+}(\lambda)\right] .
\end{aligned}
$$

Since $\phi^{(r)}(\lambda)$ is an even function and $\lambda=\lambda_{0}^{(r)}$ give the zero points, the above equations for $\eta^{(r)}(\lambda)$ can be simplified to become

$$
\begin{aligned}
& \eta^{(1)}(\lambda) \approx \int_{|\lambda|>\lambda_{0}^{(1)}} h\left(\lambda-\lambda^{\prime}\right) \eta^{(1)}\left(\lambda^{\prime}\right) \mathrm{d} \lambda^{\prime}+\int_{|\lambda|>\lambda_{0}^{(2)}} h\left(\lambda-\lambda^{\prime}\right) \eta^{(2)}\left(\lambda^{\prime}\right) \mathrm{d} \lambda^{\prime}+E_{h}^{(1)}(\lambda)+E_{g}^{(2)}(\lambda), \\
& \eta^{(2)}(\lambda) \approx \int_{|\lambda|>\lambda_{0}^{(2)}} h\left(\lambda-\lambda^{\prime}\right) \eta^{(2)}\left(\lambda^{\prime}\right) \mathrm{d} \lambda^{\prime}+\int_{|\lambda|>\lambda_{0}^{(1)}} h\left(\lambda-\lambda^{\prime}\right) \eta^{(1)}\left(\lambda^{\prime}\right) \mathrm{d} \lambda^{\prime}+E_{h}^{(2)}(\lambda)+E_{g}^{(1)}(\lambda),
\end{aligned}
$$


where the approximation

$$
\begin{aligned}
f *[T \ln (1+ & \left.\left.\mathrm{e}^{\left(\phi^{(r)}(\lambda)+\eta^{(r)}(\lambda)\right) / T}\right)-\phi^{(1)+}(\lambda)\right] \\
= & \int_{|\lambda|>\lambda_{0}^{(r)}} \mathrm{d} \lambda^{\prime} f\left(\lambda-\lambda^{\prime}\right)\left[T \ln \left(1+\mathrm{e}^{\left(\phi^{(r)}(\lambda)+\eta^{(r)}(\lambda)\right) / T}\right)-\phi^{(1)}(\lambda)\right] \\
& +\int_{-\lambda_{0}^{(r)}}^{\lambda_{0}^{(r)}} f\left(\lambda-\lambda^{\prime}\right) T \ln \left(1+\mathrm{e}^{\left(\phi^{(r)}(\lambda)+\eta^{(r)}(\lambda)\right) / T}\right) \\
\approx & \int_{|\lambda|>\lambda_{0}^{(r)}} \mathrm{d} \lambda^{\prime} f\left(\lambda-\lambda^{\prime}\right) \eta^{(r)}\left(\lambda^{\prime}\right)+E_{f}^{(r)}(\lambda) .
\end{aligned}
$$

has been made. Here $E_{f}^{(r)}(\lambda)$ stands for the integral

$$
E_{f}^{(r)}(\lambda):=\int_{-\infty}^{\infty} f\left(\lambda-\lambda^{\prime}\right) T \ln \left(1+\mathrm{e}^{\left|\phi^{(r)}(\lambda)\right| / T}\right)
$$

and $f$ represents the subscripts $h$ and $g$. For $T \rightarrow 0$, the major contribution to the integral is from the regions near the zero points of $\phi^{(r)}(\lambda)$, i.e. $\lambda= \pm \lambda_{0}^{(r)}$. Therefore, the expansion of $\phi^{(r)}(\lambda)$ around $\lambda= \pm \lambda_{0}^{(r)}$ is

$$
\phi^{(r)}(\lambda)=t^{(r)}\left(\lambda-\lambda_{0}^{(r)}\right)+O\left[\left(\lambda-\lambda_{0}^{(r)}\right)^{2}\right]
$$

where

$$
t^{(r)}:=\left.\frac{\mathrm{d} \phi^{(r)}}{\mathrm{d} \lambda}\right|_{\lambda=\lambda_{0}^{(r)}} .
$$

Then the leading term of $E_{f}^{(r)}(\lambda)$ becomes

$$
\begin{aligned}
E_{f}^{(r)}(\lambda) & \approx \frac{2 T^{2}}{t^{(r)}}\left[f\left(\lambda-\lambda_{0}^{(r)}\right)+f\left(\lambda+\lambda_{0}^{(r)}\right)\right] \int_{0}^{\infty} \mathrm{d} x \ln \left(1+\mathrm{e}^{-x}\right) \\
& =\frac{\pi^{2} T^{2}}{6 t^{(r)}}\left[f\left(\lambda-\lambda_{0}^{(r)}\right)+f\left(\lambda+\lambda_{0}^{(r)}\right)\right] .
\end{aligned}
$$

Substituting these results into (34), we obtain the equations for $\phi^{(r)}$ and $\eta^{(r)}$, namely

$$
\phi^{(1)}(\lambda)=H-2 \pi P s_{1}(\lambda)+h * \phi^{(1)+}(\lambda)+g * \phi^{(2)+}(\lambda)
$$

$\phi^{(2)}(\lambda)=H-2 \pi P s_{2}(\lambda)+h * \phi^{(2)+}(\lambda)+g * \phi^{(1)+}(\lambda)$

$$
\begin{gathered}
\eta^{(1)}(\lambda) \approx \int_{|\lambda|>\lambda_{0}^{(1)}} h\left(\lambda-\lambda^{\prime}\right) \eta^{(1)}\left(\lambda^{\prime}\right) \mathrm{d} \lambda^{\prime}+\int_{|\lambda|>\lambda_{0}^{(1)}} h\left(\lambda-\lambda^{\prime}\right) \eta^{(2)}\left(\lambda^{\prime}\right) \mathrm{d} \lambda^{\prime} \\
+\frac{\pi^{2} T^{2}}{6 t^{(1)}}\left[h\left(\lambda-\lambda_{0}^{(1)}\right)+h\left(\lambda+\lambda_{0}^{(1)}\right)\right] \\
+\frac{\pi^{2} T^{2}}{6 t^{(2)}}\left[g\left(\lambda-\lambda_{0}^{(1)}\right)+g\left(\lambda+\lambda_{0}^{(1)}\right)\right]
\end{gathered}
$$

$$
\begin{gathered}
\eta^{(2)}(\lambda) \approx \int_{|\lambda|>\lambda_{0}^{(2)}} h\left(\lambda-\lambda^{\prime}\right) \eta^{(2)}\left(\lambda^{\prime}\right) \mathrm{d} \lambda^{\prime}+\int_{|\lambda|>\lambda_{0}^{(2)}} h\left(\lambda-\lambda^{\prime}\right) \eta^{(1)}\left(\lambda^{\prime}\right) \mathrm{d} \lambda^{\prime} \\
+\frac{\pi^{2} T^{2}}{6 t^{(2)}}\left[h\left(\lambda-\lambda_{0}^{(2)}\right)+h\left(\lambda+\lambda_{0}^{(2)}\right)\right] \\
+\frac{\pi^{2} T^{2}}{6 t^{(1)}}\left[g\left(\lambda-\lambda_{0}^{(2)}\right)+g\left(\lambda+\lambda_{0}^{(2)}\right)\right] .
\end{gathered}
$$


When $H \rightarrow 0, \lambda_{0}^{(r)} \rightarrow \infty,(40)$ and (41) can be simplified to

$$
\phi^{(r)}(\lambda)=H-2 \pi P s_{r}(\lambda)
$$

Since $\phi^{(r)}\left(\lambda_{0}^{(r)}\right)=0, s_{r}\left(\lambda_{0}^{(r)}\right)$ can be written as

$$
s_{r}\left(\lambda_{0}^{(r)}\right)=\frac{H}{2 \pi P} .
$$

From (13) and (14) we find that in the limit $\lambda_{0}^{(r)} \gg 1$,

$$
s_{r}\left(\lambda_{0}^{(r)}\right)=\frac{1}{\sqrt{3} c} \mathrm{e}^{-2 \pi \lambda_{0}^{(r)} / 3 c}+O\left(\frac{1}{c^{2}}\right) .
$$

Hence from both equations (45) and (46) we obtain

$$
\lambda_{0}^{(r)}=-\frac{3 c}{2 \pi}\left[\ln \left(\frac{\sqrt{3} c}{2 \pi} H\right)+\ln \kappa^{(r)}\right]
$$

where $\kappa^{(r)} \approx 1$ is an integral constant.

Instead of $\phi^{(r)}(\lambda)$ and $\eta^{(r)}(\lambda)$, it is easier to work with the functions

$$
\begin{aligned}
& S^{(r)}(\lambda)= \begin{cases}\mathrm{e}^{2 \pi \lambda_{0}^{(r)} / 3 c} \kappa^{(r)} \phi^{(r)}\left(\lambda+\lambda_{0}^{(r)}\right), & \text { for } \lambda>0 ; \\
0, & \text { for } \lambda<0 .\end{cases} \\
& T^{(r)}(\lambda)= \begin{cases}\frac{6 \mathrm{e}^{-2 \pi \lambda_{0}^{(r)} / 3 c}}{\pi^{2} T^{2} \kappa^{(r)}} \eta^{(r)}\left(\lambda+\lambda_{0}^{(r)}\right), & \text { for } \lambda>0 ; \\
0, & \text { for } \lambda<0 .\end{cases}
\end{aligned}
$$

Note that $\phi^{(r)}(\lambda)>0$ for $|\lambda|>\lambda_{0}^{(r)}$. Instead, we shift the integration variables so that they run from 0 to $\infty$. Observe that $h\left(\lambda+2 \lambda_{0}^{(r)}\right)$ and $g\left(\lambda+\lambda_{0}^{(1)}+\lambda_{0}^{(2)}\right)$ vanish as $H \rightarrow 0$ $\left(\lambda_{0}^{(r)} \rightarrow \infty\right)$ for finite $\lambda$. The functions $g\left(\lambda+\lambda_{0}^{(1)}-\lambda_{0}^{(2)}\right)$ and $g\left(\lambda-\lambda_{0}^{(1)}+\lambda_{0}^{(2)}\right)$ remain finite. Equations (40)-(43) in the form of the newly introduced functions are

$$
\begin{aligned}
S^{(1)}(\lambda)= & \frac{2 \pi P}{\sqrt{3} c}\left(1-\kappa^{(1)} \mathrm{e}^{-2 \pi \lambda / 3 c}\right)+\int_{0}^{\infty} h\left(\lambda-\lambda^{\prime}\right) S^{(1)}\left(\lambda^{\prime}\right) \mathrm{d} \lambda^{\prime} \\
& +\int_{0}^{\infty} g\left(\lambda+\lambda_{0}^{(1)}-\lambda^{\prime}-\lambda_{0}^{(2)}\right) S^{(2)}\left(\lambda^{\prime}\right) \mathrm{d} \lambda^{\prime}, \\
T^{(1)}(\lambda)= & \frac{h(\lambda)}{S^{(1) \prime}(0)}+\frac{g\left(\lambda+\lambda_{0}^{(1)}-\lambda_{0}^{(2)}\right)}{S^{(2) \prime}(0)}+\int_{0}^{\infty} h\left(\lambda-\lambda^{\prime}\right) T^{(1)}\left(\lambda^{\prime}\right) \mathrm{d} \lambda^{\prime} \\
& +\int_{0}^{\infty} g\left(\lambda+\lambda_{0}^{(1)}-\lambda^{\prime}-\lambda_{0}^{(2)}\right) T^{(2)}\left(\lambda^{\prime}\right) \mathrm{d} \lambda^{\prime}, \\
S^{(2)}(\lambda)= & \frac{2 \pi P}{\sqrt{3} c}\left(1-\kappa^{(2)} \mathrm{e}^{-2 \pi \lambda / 3 c}\right)+\int_{0}^{\infty} h\left(\lambda-\lambda^{\prime}\right) S^{(2)}\left(\lambda^{\prime}\right) \mathrm{d} \lambda^{\prime} \\
& +\int_{0}^{\infty} g\left(\lambda+\lambda_{0}^{(2)}-\lambda^{\prime}-\lambda_{0}^{(1)}\right) S^{(1)}\left(\lambda^{\prime}\right) \mathrm{d} \lambda^{\prime}, \\
T^{(2)}(\lambda)= & \frac{h(\lambda)}{S^{(2) \prime}(0)}+\frac{g\left(\lambda+\lambda_{0}^{(2)}-\lambda_{0}^{(1)}\right)}{S^{(1) \prime}(0)}+\int_{0}^{\infty} h\left(\lambda-\lambda^{\prime}\right) T^{(2)}\left(\lambda^{\prime}\right) \mathrm{d} \lambda^{\prime} \\
& +\int_{0}^{\infty} g\left(\lambda+\lambda_{0}^{(2)}-\lambda^{\prime}-\lambda_{0}^{(1)}\right) T^{(1)}\left(\lambda^{\prime}\right) \mathrm{d} \lambda^{\prime} .
\end{aligned}
$$


Writing the equations in standard Wiener-Hopf form for $-\infty<\lambda<\infty$ gives

$$
\begin{array}{r}
S^{(1)}(\lambda)=f_{S}^{(1)}(\lambda)+b_{S}^{(1)}(\lambda)+\int_{-\infty}^{\infty} h\left(\lambda-\lambda^{\prime}\right) S^{(1)}\left(\lambda^{\prime}\right) \mathrm{d} \lambda^{\prime} \\
+\int_{-\infty}^{\infty} g\left(\lambda+\lambda_{0}^{(1)}-\lambda^{\prime}-\lambda_{0}^{(2)}\right) S^{(2)}\left(\lambda^{\prime}\right) \mathrm{d} \lambda^{\prime}, \\
T^{(1)}(\lambda)=f_{T}^{(1)}(\lambda)+b_{T}^{(1)}(\lambda)+\int_{-\infty}^{\infty} h\left(\lambda-\lambda^{\prime}\right) T^{(1)}\left(\lambda^{\prime}\right) \mathrm{d} \lambda^{\prime} \\
+\int_{-\infty}^{\infty} g\left(\lambda+\lambda_{0}^{(1)}-\lambda^{\prime}-\lambda_{0}^{(2)}\right) T^{(2)}\left(\lambda^{\prime}\right) \mathrm{d} \lambda^{\prime}, \\
S^{(2)}(\lambda)=f_{S}^{(2)}(\lambda)+b_{S}^{(2)}(\lambda)+\int_{-\infty}^{\infty} h\left(\lambda-\lambda^{\prime}\right) S^{(2)}\left(\lambda^{\prime}\right) \mathrm{d} \lambda^{\prime} \\
+\int_{-\infty}^{\infty} g\left(\lambda+\lambda_{0}^{(2)}-\lambda^{\prime}-\lambda_{0}^{(1)}\right) S^{(1)}\left(\lambda^{\prime}\right) \mathrm{d} \lambda^{\prime}, \\
T^{(2)}(\lambda)=f_{T}^{(2)}(\lambda)+b_{T}^{(2)}(\lambda)+\int_{-\infty}^{\infty} h\left(\lambda-\lambda^{\prime}\right) T^{(2)}\left(\lambda^{\prime}\right) \mathrm{d} \lambda^{\prime} \\
+\int_{-\infty}^{\infty} g\left(\lambda+\lambda_{0}^{(2)}-\lambda^{\prime}-\lambda_{0}^{(1)}\right) T^{(1)}\left(\lambda^{\prime}\right) \mathrm{d} \lambda^{\prime},
\end{array}
$$

where

$$
\begin{aligned}
& f_{S}^{(r)}(\lambda)= \begin{cases}\frac{2 \pi P}{\sqrt{3} c}\left(1-\kappa^{(r)} \mathrm{e}^{-2 \pi \lambda / 3 c}\right), & \text { for } \lambda>0 \\
0, & \text { for } \lambda<0 .\end{cases} \\
& b_{S}^{(1)}(\lambda)= \begin{cases}0, & \text { for } \lambda>0 \\
-h * S^{(1)}(\lambda)-g * S^{(2)}\left(\lambda+\lambda_{0}^{(1)}-\lambda_{0}^{(2)}\right), & \text { for } \lambda<0 .\end{cases} \\
& b_{S}^{(2)}(\lambda)= \begin{cases}0, & \text { for } \lambda>0 \\
-h * S^{(2)}(\lambda)-g * S^{(1)}\left(\lambda+\lambda_{0}^{(1)}-\lambda_{0}^{(2)}\right), & \text { for } \lambda<0 .\end{cases} \\
& f_{T}^{(1)}(\lambda)= \begin{cases}\frac{h(\lambda)}{S^{(1) \prime}(0)}+\frac{g\left(\lambda+\lambda_{0}^{(1)}-\lambda_{0}^{(2)}\right)}{S^{(2) \prime}(0)}, & \text { for } \lambda>0 ; \\
0, & \text { for } \lambda<0 .\end{cases} \\
& f_{T}^{(2)}(\lambda)= \begin{cases}\frac{h(\lambda)}{S^{(2) \prime}(0)}+\frac{g\left(\lambda+\lambda_{0}^{(1)}-\lambda_{0}^{(2)}\right)}{S^{(1) \prime}(0)}, & \text { for } \lambda>0 \\
0, & \text { for } \lambda<0 .\end{cases} \\
& b_{T}^{(1)}(\lambda)= \begin{cases}0, & \text { for } \lambda>0 \\
-h * T^{(1)}(\lambda)-g * T^{(2)}\left(\lambda+\lambda_{0}^{(1)}-\lambda_{0}^{(2)}\right), & \text { for } \lambda<0 .\end{cases} \\
& b_{T}^{(2)}(\lambda)= \begin{cases}0, & \text { for } \lambda>0 \\
-h * T^{(2)}(\lambda)-g * T^{(1)}\left(\lambda+\lambda_{0}^{(1)}-\lambda_{0}^{(2)}\right), & \text { for } \lambda<0\end{cases}
\end{aligned}
$$


The equations (54)-(57) can be solved via Fourier transform. Define the Fourier coefficients of $S^{(r)}(\lambda)$ and $T^{(r)}(\lambda)$ as

$$
\begin{aligned}
& \hat{S}^{(r)}(\omega)=\int_{-\infty}^{\infty} \mathrm{d} \lambda \mathrm{e}^{\mathrm{i} \lambda \omega} S^{(r)}(\lambda), \\
& \hat{T}^{(r)}(\omega)=\int_{-\infty}^{\infty} \mathrm{d} \lambda \mathrm{e}^{\mathrm{i} \lambda \omega} T^{(r)}(\lambda) .
\end{aligned}
$$

The functions $\hat{S}^{(r)}(\omega)$ and $\hat{T}^{(r)}(\omega)$ are analytic on the upper-half-plane.

For simplicity, we write equations (54)-(57) in matrix form

$$
\begin{aligned}
& \mathbf{S}(\lambda)=\mathbf{f}_{\mathbf{S}}(\lambda)+\mathbf{b}_{\mathbf{S}}(\lambda)+\int_{-\infty}^{\infty} \mathbf{K}\left(\lambda-\lambda^{\prime}\right) \mathbf{S}\left(\lambda^{\prime}\right) \mathrm{d} \lambda^{\prime}, \\
& \mathbf{T}(\lambda)=\mathbf{f}_{\mathbf{T}}(\lambda)+\mathbf{b}_{\mathbf{T}}(\lambda)+\int_{-\infty}^{\infty} \mathbf{K}\left(\lambda-\lambda^{\prime}\right) \mathbf{T}\left(\lambda^{\prime}\right) \mathrm{d} \lambda^{\prime},
\end{aligned}
$$

where

$$
\begin{aligned}
& \mathbf{S}(\lambda)=\left(\begin{array}{l}
S^{(1)}(\lambda) \\
S^{(2)}(\lambda)
\end{array}\right), \quad \mathbf{T}(\lambda)=\left(\begin{array}{l}
T^{(1)}(\lambda) \\
T^{(2)}(\lambda)
\end{array}\right) \\
& \mathbf{f}_{\mathbf{S}}(\lambda)=\left(\begin{array}{l}
f_{S}^{(1)}(\lambda) \\
f_{S}^{(2)}(\lambda)
\end{array}\right), \quad \mathbf{b}_{\mathbf{S}}(\lambda)=\left(\begin{array}{l}
b_{S}^{(1)}(\lambda) \\
b_{S}^{(2)}(\lambda)
\end{array}\right), \\
& \mathbf{f}_{\mathbf{T}}(\lambda)=\left(\begin{array}{l}
f_{T}^{(1)}(\lambda) \\
f_{T}^{(2)}(\lambda)
\end{array}\right), \quad \mathbf{b}_{\mathbf{T}}(\lambda)=\left(\begin{array}{l}
b_{T}^{(1)}(\lambda) \\
b_{T}^{(2)}(\lambda)
\end{array}\right), \\
& \mathbf{K}(\lambda)=\left(\begin{array}{cc}
h(\lambda) & g\left(\lambda+\lambda_{0}^{(1)}-\lambda_{0}^{(2)}\right) \\
g\left(\lambda+\lambda_{0}^{(2)}-\lambda_{0}^{(1)}\right) & h(\lambda)
\end{array}\right) .
\end{aligned}
$$

We denote Fourier transforms of $\mathbf{S}(\lambda)$ and $\mathbf{T}(\lambda)$ as

$$
\begin{aligned}
& \hat{\mathbf{S}}(\omega)=\int_{-\infty}^{\infty} \mathrm{d} \lambda \mathrm{e}^{\mathrm{i} \lambda \omega} \mathbf{S}(\lambda), \\
& \hat{\mathbf{T}}(\omega)=\int_{-\infty}^{\infty} \mathrm{d} \lambda \mathrm{e}^{\mathrm{i} \lambda \omega} \mathbf{T}(\lambda),
\end{aligned}
$$

which represents a Fourier transformation on each of the matrix components.

Thus the Wiener-Hopf equations for $\mathbf{S}(\lambda)$ and $\mathbf{T}(\lambda)$ in Fourier space are

$$
\begin{aligned}
& \hat{\mathbf{S}}(\omega)=\hat{\mathbf{f}}_{\mathbf{S}}(\omega)+\hat{\mathbf{b}}_{\mathbf{S}}(\omega)+\hat{\mathbf{K}}(\omega) \hat{\mathbf{S}}(\omega), \\
& \hat{\mathbf{T}}(\omega)=\hat{\mathbf{f}}_{\mathbf{T}}(\omega)+\hat{\mathbf{b}}_{\mathbf{T}}(\omega)+\hat{\mathbf{K}}(\omega) \hat{\mathbf{T}}(\omega),
\end{aligned}
$$

where

$$
\hat{\mathbf{K}}(\omega)=\left(\begin{array}{cc}
\hat{h}(\omega) & \mathrm{e}^{-\mathrm{i} \omega\left(\lambda_{0}^{(1)}-\lambda_{0}^{(2)}\right)} \hat{g}(\omega) \\
\mathrm{e}^{\mathrm{i} \omega\left(\lambda_{0}^{(1)}-\lambda_{0}^{(2)}\right)} \hat{g}(\omega) & \hat{h}(\omega)
\end{array}\right) .
$$

Note that when $\omega$ and $\lambda_{0}^{(r)}$ are real, the kernel $\hat{\mathbf{K}}(\omega)$ is Hermitian, i.e.

$$
\hat{\mathbf{K}}^{\dagger}(\omega)=\hat{\mathbf{K}}(\omega)
$$


Equations (75) and (76) can also be written as

$$
\begin{aligned}
& \hat{\mathbf{S}}(\omega)=(\mathbf{I}-\hat{\mathbf{K}}(\omega))^{-\mathbf{1}}\left(\hat{\mathbf{f}}_{\mathbf{S}}(\omega)+\hat{\mathbf{b}}_{\mathbf{S}}(\omega)\right), \\
& \hat{\mathbf{T}}(\omega)=(\mathbf{I}-\hat{\mathbf{K}}(\omega))^{-\mathbf{1}}\left(\hat{\mathbf{f}}_{\mathbf{T}}(\omega)+\hat{\mathbf{b}}_{\mathbf{T}}(\omega)\right) .
\end{aligned}
$$

Since the function $(\mathbf{I}-\hat{\mathbf{K}}(\omega))^{-1}$ is nonsingular, Hermitian and positive definite at $\omega=0$, it is also positive definite for $-\infty<\omega<\infty$. From theorem 8.2 of Gohberg and Krein [52], the function can be factorized as follows:

$$
(\mathbf{I}-\hat{\mathbf{K}}(\omega))^{-1}=\mathbf{G}_{+}(\omega) \mathbf{G}_{-}(\omega),
$$

where each element of the $2 \times 2$ matrix $\mathbf{G}_{+}(\omega)\left(\mathbf{G}_{-}(\omega)\right)$ is analytic on the upper-half (lowerhalf) plane. The limit $\mathbf{G}_{ \pm}(\omega)$ is chosen to satisfy the condition

$$
\mathbf{G}_{ \pm}(\infty)=\mathbf{I}
$$

where $\mathbf{I}$ is the identity matrix. From (78), it follows that

$$
\mathbf{G}_{+}(-\omega)=\mathbf{G}_{-}^{\mathbf{T}}(\omega),
$$

where $\mathbf{A}^{T}$ represents the transpose of the matrix $\mathbf{A}$.

In appendices A and B, we have worked out the solutions to equations (79) and (80) in detail. We showed in equations (A.9) and (B.9) that their solutions are

$$
\hat{\mathbf{S}}(\omega)=\mathrm{i} \frac{2 \pi P}{\sqrt{3} c}\left(\frac{1}{\omega+\mathrm{i} \epsilon}-\frac{1}{\omega+2 \pi \mathrm{i} / 3 c}\right) \mathbf{G}_{+}(\omega) \mathbf{G}_{-}(0)\left(\begin{array}{l}
1 \\
1
\end{array}\right)
$$

and

$$
\hat{\mathbf{T}}(\omega)=\left(\mathbf{G}_{+}(\omega)-\mathbf{I}\right) \mathbf{V}
$$

where $\mathbf{V}$ is a $2 \times 1$ matrix given in equation (B.5).

\section{Universal low-temperature properties and spin-charge separation}

To solve the TBA equations (6) and (10), we must find a way to express them involving the functions $\hat{S}$ and $\hat{T}$. Let us rewrite the dressed energy from the first set of TBA equations (6) as

$$
\begin{aligned}
\varepsilon(k)= & k^{2}-\mu-2 \pi P s_{1} * a_{1}(k)-T \int_{-\infty}^{\infty} s_{1}(k-\lambda) \ln \left(1+\mathrm{e}^{\phi_{1}^{(1)} / T}\right) \mathrm{d} \lambda \\
& -T \int_{-\infty}^{\infty} s_{2}(k-\lambda) \ln \left(1+\mathrm{e}^{\phi_{1}^{(2)} / T}\right) \mathrm{d} \lambda \\
\approx & k^{2}-\mu-2 \pi P s_{1} * a_{1}(k)-T \int_{|\lambda|>\lambda_{0}^{(1)}} s_{1}(k-\lambda) \ln \left(1+\mathrm{e}^{\phi_{1}^{(1)} / T}\right) \mathrm{d} \lambda \\
& -T \int_{-\lambda_{0}^{(1)}}^{\lambda_{0}^{(1)}} s_{1}(k-\lambda) \ln \left(1+\mathrm{e}^{\phi_{1}^{(1)} / T}\right) \mathrm{d} \lambda-T \int_{|\lambda|>\lambda_{0}^{(2)}} s_{2}(k-\lambda) \ln \left(1+\mathrm{e}^{\phi_{1}^{(2)} / T}\right) \mathrm{d} \lambda \\
& -T \int_{-\lambda_{0}^{(2)}}^{\lambda_{0}^{(2)}} s_{2}(k-\lambda) \ln \left(1+\mathrm{e}^{\phi_{1}^{(2)} / T}\right) \mathrm{d} \lambda \\
\approx & k^{2}-\mu-2 \pi P s_{1} * a_{1}(k)-T \int_{|\lambda|>\lambda_{0}^{(1)}} s_{1}(k-\lambda) \ln \left(1+\mathrm{e}^{\phi_{1}^{(1)} / T}\right) \mathrm{d} \lambda \\
& -T \int_{|\lambda|>\lambda_{0}^{(2)}} s_{2}(k-\lambda) \ln \left(1+\mathrm{e}^{\phi_{1}^{(2)} / T}\right) \mathrm{d} \lambda-\sum_{r=1}^{2} \frac{\pi^{2} T^{2} \kappa^{(r)}}{3 \sqrt{3} c S^{(r) \prime}(0)}
\end{aligned}
$$


where we evaluated the integration between the Fermi points as

$$
\begin{aligned}
\sum_{r=1}^{2} T \int_{-\lambda_{0}^{(r)}}^{\lambda_{0}^{(r)}} s_{r}(k-\lambda) \ln \left(1+\mathrm{e}^{\phi_{1}^{(r)} / T}\right) \mathrm{d} \lambda & \approx \sum_{r=1}^{2} T \int_{-\infty}^{\infty} s_{r}(k-\lambda) \ln \left(1+\mathrm{e}^{-\left|\phi_{1}^{(r)}\right| / T}\right) \mathrm{d} \lambda \\
& \approx \sum_{r=1}^{2} \frac{\pi^{2} T^{2}}{6 t^{(r)}}\left[s_{r}\left(k-\lambda_{0}^{(r)}\right)+s_{r}\left(k+\lambda_{0}^{(r)}\right)\right] \\
& \approx \sum_{r=1}^{2} \frac{\pi^{2} T^{2}}{3 \sqrt{3} c t^{(r)}} \mathrm{e}^{-2 \pi \lambda_{0}^{(r)} / 3 c} \\
& =\sum_{r=1}^{2} \frac{\pi^{2} T^{2} \kappa^{(r)}}{3 \sqrt{3} c S^{(r) \prime}(0)}
\end{aligned}
$$

From the third line to the fourth line, we made use of the fact that

$$
S^{(r) \prime}(0)=\mathrm{e}^{2 \pi \lambda_{0}^{(r)} / 3 c} t^{(r)} \kappa^{(r)} .
$$

The other two integrals can be simplified as

$$
\begin{aligned}
\int_{\lambda_{0}^{(r)}}^{\infty} s_{r}(k-\lambda) & {\left[\phi^{(r)}+\eta^{(r)}\right] \mathrm{d} \lambda=\int_{0}^{\infty} s_{r}\left(k-\lambda-\lambda_{0}^{(r)}\right) } \\
& \times\left[\frac{\mathrm{e}^{-2 \pi \lambda_{0}^{(r)} / 3 c}}{\kappa^{(r)}} S^{(r)}(\lambda)+\frac{\pi^{2} T^{2} \kappa^{(r)}}{6 \mathrm{e}^{-2 \pi \lambda_{0}^{(r)} / 3 c}} T^{(r)}(\lambda)\right] \mathrm{d} \lambda \\
\approx & \int_{0}^{\infty} \frac{\mathrm{e}^{2 \pi\left(k-\lambda-2 \lambda_{0}^{(r)}\right) / 3 c}}{\sqrt{3} c \kappa^{(r)}} S^{(r)}(\lambda) \mathrm{d} \lambda+\int_{0}^{\infty} \frac{\pi^{2} T^{2} \kappa^{(r)} \mathrm{e}^{2 \pi(k-\lambda) / 3 c}}{6 \sqrt{3} c} T^{(r)}(\lambda) \mathrm{d} \lambda \\
= & \int_{0}^{\infty} \frac{\sqrt{3} c H^{2} \kappa^{(r)}}{4 \pi^{2} P^{2}} \mathrm{e}^{2 \pi(k-\lambda) / 3 c} S^{(r)}(\lambda) \mathrm{d} \lambda+\int_{0}^{\infty} \frac{\pi^{2} T^{2} \kappa^{(r)} \mathrm{e}^{2 \pi(k-\lambda) / 3 c}}{6 \sqrt{3} c} T^{(r)}(\lambda) \mathrm{d} \lambda \\
& =\int_{0}^{\infty} \frac{\sqrt{3} c H^{2} \kappa^{(r)}}{8 \pi^{3} P^{2}} \mathrm{e}^{2 \pi(k-\lambda) / 3 c} \mathrm{e}^{-\mathrm{i} \omega \lambda} \hat{S}^{(r)}(\omega) \mathrm{d} \omega \mathrm{d} \lambda \\
& +\int_{0}^{\infty} \int_{-\infty}^{\infty} \frac{\pi T^{2} \kappa^{(r)}}{12 \sqrt{3} c} \mathrm{e}^{2 \pi(k-\lambda) / 3 c} \mathrm{e}^{-\mathrm{i} \omega \lambda} \hat{T}^{(r)}(\omega) \mathrm{d} \omega \mathrm{d} \lambda \\
\approx & \int_{-\infty}^{\infty} \frac{\sqrt{3} c H^{2} \kappa^{(r)}}{8 \pi^{3} P^{2}} \frac{\hat{S}^{(r)}(\omega)}{\mathrm{i} \omega+2 \pi / 3 c} \mathrm{e}^{2 \pi k / 3 c} \mathrm{~d} \omega+\int_{-\infty}^{\infty} \frac{\pi T^{2} \kappa^{(r)}}{12 \sqrt{3} c} \frac{\hat{T}^{(r)}(\omega)}{\mathrm{i} \omega+2 \pi / 3 c} \mathrm{e}^{2 \pi k / 3 c} \mathrm{~d} \omega \\
= & \frac{\sqrt{3} c H^{2} \kappa^{(r)}}{4 \pi^{2} P^{2}} \hat{S}^{(r)}\left(\frac{2 \pi \mathrm{i}}{3 c}\right)+\frac{\pi^{2} T^{2} \kappa^{(r)}}{6 \sqrt{3} c} \hat{T}^{(r)}\left(\frac{2 \pi \mathrm{i}}{3 c}\right) \cdot
\end{aligned}
$$

Thus the dressed energy is

$$
\begin{aligned}
\varepsilon(k) \approx & k^{2}-\mu-2 \pi P s_{1} * a_{1}(k)-2 \sum_{r=1}^{2} \int_{\lambda_{0}^{(r)}}^{\infty} s_{r}(k-\lambda)\left[\phi^{(r)}+\eta^{(r)}\right] \mathrm{d} \lambda-\sum_{r=1}^{2} \frac{\pi^{2} T^{2} \kappa^{(r)}}{3 \sqrt{3} c S^{(r) \prime}(0)} \\
= & k^{2}-\mu-2 \pi P s_{1} * a_{1}(k) \\
& -\sum_{r=1}^{2}\left[\frac{\sqrt{3} c H^{2} \kappa^{(r)}}{2 \pi^{2} P^{2}} \hat{S}^{(r)}\left(\frac{2 \pi i}{3 c}\right)+\frac{\pi^{2} T^{2} \kappa^{(r)}}{3 \sqrt{3} c} \hat{T}^{(r)}\left(\frac{2 \pi i}{3 c}\right)+\frac{\pi^{2} T^{2} \kappa^{(r)}}{3 \sqrt{3} c S^{(r) \prime}(0)}\right]
\end{aligned}
$$

From (A.9), $\hat{\mathbf{S}}(2 \pi \mathrm{i} / 3 c)$ is expressed as

$$
\hat{\mathbf{S}}\left(\frac{2 \pi \mathrm{i}}{3 c}\right)=\frac{\sqrt{3}}{2} P \mathbf{G}_{+}\left(\frac{2 \pi \mathbf{i}}{3 c}\right) \mathbf{G}_{-}(0)\left(\begin{array}{l}
1 \\
1
\end{array}\right) .
$$


To evaluate the sum $\sum_{r=1}^{2} \kappa^{(r)} \hat{S}^{(r)}(2 \pi \mathrm{i} / 3 c)$, we only need to consider the matrix product

$$
\begin{aligned}
& \left(\kappa^{(1)} \quad \kappa^{(2)}\right) \hat{\mathbf{S}}\left(\frac{2 \pi \mathrm{i}}{3 c}\right)=\frac{\sqrt{3}}{2} P\left(\kappa^{(1)} \kappa^{(2)}\right) \mathbf{G}_{+}\left(\frac{2 \pi \mathrm{i}}{3 c}\right) \mathbf{G}_{-}(0)\left(\begin{array}{l}
1 \\
1
\end{array}\right) \\
& =\frac{\sqrt{3}}{2} P\left(\begin{array}{ll}
1 & 1
\end{array}\right) \mathbf{G}_{+}(0) \mathbf{G}_{-}(0)\left(\begin{array}{l}
1 \\
1
\end{array}\right) \\
& =\sqrt{3} P \text {. }
\end{aligned}
$$

We made use of equations (83) and (A.8) to derive the second line from the first line. The term $\mathbf{G}_{+}(0) \mathbf{G}_{-}(0)$ was explicitly derived from its original definition (81). To do that, we needed to find $\hat{\mathbf{K}}(0)$. Since

$$
\hat{s}_{1}(0)=\int_{-\infty}^{\infty} s_{1}(x) \mathrm{d} x=\frac{2}{3}, \quad \hat{s}_{2}(0)=\int_{-\infty}^{\infty} s_{2}(x) \mathrm{d} x=\frac{1}{3},
$$

hence

$$
\mathbf{G}_{+}(0) \mathbf{G}_{-}(0)=(\mathbf{I}-\hat{\mathbf{K}}(0))^{-1}=\left(\begin{array}{cc}
2 & -1 \\
-1 & 2
\end{array}\right) .
$$

The next step is to evaluate the $\sum_{r=1}^{2} \kappa^{(r)}\left[\hat{T}^{(r)}(2 \pi \mathrm{i} / 3 c)+1 / S^{(r) \prime}(0)\right]$. Similar to the previous calculation, it is easier to work with the matrix representations. Doing so yields

$$
\begin{aligned}
\left(\kappa^{(1)} \quad \kappa^{(2)}\right)\left(\hat{\mathbf{T}}\left(\frac{2 \pi \mathrm{i}}{3 c}\right)+\mathbf{V}\right) & =\left(\kappa^{(1)} \quad \kappa^{(2)}\right) \mathbf{G}_{+}\left(\frac{2 \pi \mathrm{i}}{3 c}\right) \mathbf{V} \\
& =\left(\begin{array}{ll}
1 & 1
\end{array}\right) \mathbf{G}_{+}(0) \mathbf{V} \\
& =\frac{3 \sqrt{3} c^{2}}{4 \pi^{2} P} \mathbf{S}^{\prime \mathbf{T}}(0) \mathbf{V} \\
& =\frac{3 \sqrt{3} c^{2}}{2 \pi^{2} P}
\end{aligned}
$$

where we made use of the transpose of equation (A.11), which is

$$
\mathbf{S}^{\prime \mathbf{T}}(0)=\frac{4 \pi^{2} P}{3 \sqrt{3} c^{2}}\left(\begin{array}{ll}
1 & 1) \mathbf{G}_{+}(0)
\end{array}\right.
$$

Finally, the dressed energy can be written as

$$
\varepsilon(k) \approx k^{2}-\mu-2 \pi P s_{1} * a_{1}(k)-\frac{3 c H^{2}}{2 \pi^{2} P}-\frac{c T^{2}}{2 P} .
$$

Here

$$
\begin{aligned}
s_{1} * a_{1}(k) & =\int_{-\infty}^{\infty} s_{1}(k-\lambda) a_{1}(\lambda) \mathrm{d} \lambda \\
& \approx \int_{-\infty}^{\infty} s_{1}(\lambda) a_{1}(\lambda) \mathrm{d} \lambda \\
& =\frac{1}{6 \sqrt{3} c}+\frac{\ln 3}{2 \pi c} .
\end{aligned}
$$

Through integration by parts, equation (16) is given by

$$
P=\frac{1}{\pi} \int_{0}^{\infty} \frac{\sqrt{\varepsilon} \mathrm{d} \varepsilon}{1+\mathrm{e}^{(\varepsilon-A) / T}},
$$

where the dressed energy is rewritten in the form

$$
\varepsilon(k)=k^{2}-A(T, H)
$$


with

$$
A(T, H)=\mu+2 \pi P s_{1} * a_{1}(k)+\frac{3 c H^{2}}{2 \pi^{2} P}+\frac{c T^{2}}{2 P} .
$$

For finite temperature, the pressure can be given by polylogarithm function

$$
P=-\sqrt{\frac{1}{4 \pi}} T^{\frac{3}{2}} \operatorname{Li}_{\frac{3}{2}}\left(-\mathrm{e}^{\frac{A}{T}}\right) .
$$

This equation describes the exact low-temperature thermodynamics and thus the full phase diagram can be given in finite temperature in a much larger regime than that given by field theory. However, the low-temperature properties cannot be seen directly from the polylog functions. Therefore, we apply Sommerfeld's expansion technique for this equation and study the universal low-temperature properties. The expansion of the pressure is in terms of powers of $(T / A(T, H))$. Consider the leading order, the equation is

$$
P=\frac{2}{3 \pi} A^{\frac{3}{2}}\left[1+\frac{\pi^{2}}{8}\left(\frac{T}{A}\right)^{2}\right] .
$$

Furthermore, with the relation $n=\partial P / \partial \mu$, after the iteration, and neglecting higher order terms of $T^{2}, H^{2}$ and $1 / c$, the chemical potential $\mu$ is obtained as

$$
\begin{gathered}
\mu=n^{2} \pi^{2}[1- \\
-\frac{16 \pi n s_{1} * a_{1}(k)}{3}+\frac{9 c H^{2}}{4 n^{5} \pi^{6}}\left(1+3 \pi n s_{1} * a_{1}(k)\right) \\
\left.+\frac{3 c T^{2}}{2 n^{5} \pi^{4}}\left(1+3 \pi n s_{1} * a_{1}(k)\right)+\frac{T^{2}}{12 n^{4} \pi^{2}}\right] .
\end{gathered}
$$

Substituting (104) into (103), the pressure $P$ becomes

$$
\begin{aligned}
P=\frac{2}{3 \pi} n^{3} \pi^{3}[ & 1-6 \pi n s_{1} * a_{1}(k)+\frac{81 c H^{2}}{8 n^{5} \pi^{6}}\left(1+4 \pi n s_{1} * a_{1}(k)\right) \\
& \left.+\frac{27 c T^{2}}{8 n^{5} \pi^{4}}\left(1+4 \pi n s_{1} * a_{1}(k)\right)+\frac{T^{2}}{4 n^{4} \pi^{2}}\left(1+2 \pi n s_{1} * a_{1}(k)\right)\right] .
\end{aligned}
$$

Finally, with the relation $F=\mu n-P$, the free energy suggests a universal low-temperature behavior of TLL, namely

$$
\begin{aligned}
F= & \mu n-P \\
= & \frac{1}{3} n^{3} \pi^{2}\left(1-4 \pi n s_{1} * a_{1}(k)\right)-\frac{9 c H^{2}}{4 n^{2} \pi^{4}}\left(1+6 \pi n s_{1} * a_{1}(k)\right) \\
& -\frac{3 c T^{2}}{4 n^{2} \pi^{2}}\left(1+6 \pi n s_{1} * a_{1}(k)\right)-\frac{T^{2}}{12 n}\left(1+4 \pi n s_{1} * a_{1}(k)\right) \\
= & E_{0}-\frac{\pi T^{2}}{6}\left(\frac{C_{s}}{v_{s}}+\frac{C_{c}}{v_{c}}\right)
\end{aligned}
$$

where

$$
\begin{aligned}
E_{0} & =\frac{1}{3} n^{3} \pi^{2}\left(1-4 \pi n s_{1} * a_{1}(k)\right)-\frac{9 c H^{2}}{4 n^{2} \pi^{4}}\left(1+6 \pi n s_{1} * a_{1}(k)\right) \\
& =\frac{1}{3} n^{3} \pi^{2}\left(1-\frac{2 \pi n}{3 \sqrt{3} c}-\frac{2 n \ln 3}{c}\right)-\frac{9 c H^{2}}{4 n^{2} \pi^{4}}\left(1+\frac{\pi n}{\sqrt{3} c}+\frac{3 n \ln 3}{c}\right) \\
v_{s} & =\frac{4}{9 c} n^{2} \pi^{3}\left(1-6 \pi n s_{1} * a_{1}(k)\right) \\
& =\frac{4}{9 c} n^{2} \pi^{3}\left(1-\frac{\pi n}{\sqrt{3} c}-\frac{3 n \ln 3}{c}\right)
\end{aligned}
$$



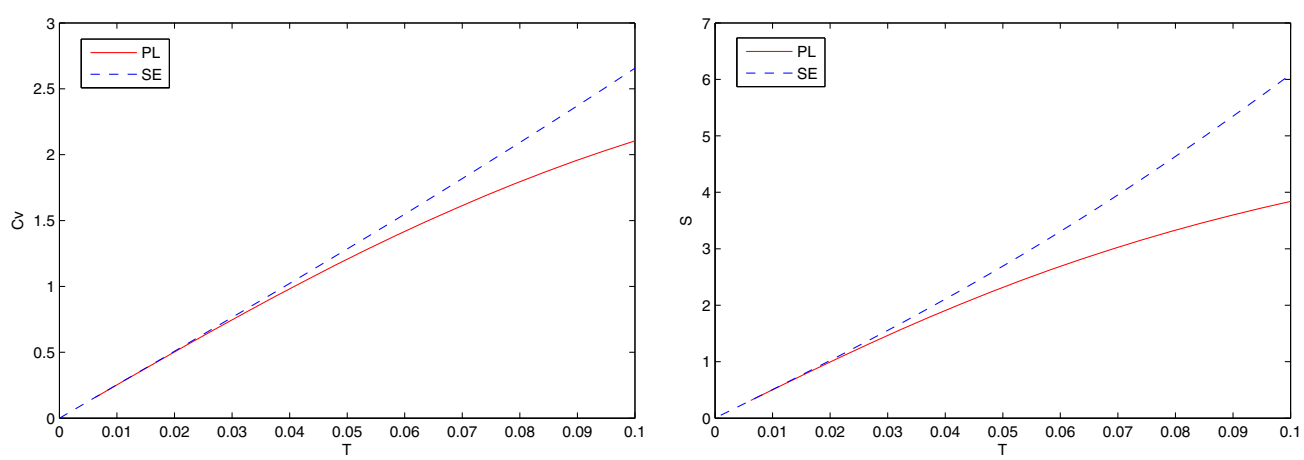

Figure 1. The left plot shows $C_{V}-T$ curves for external magnetic field $H=0.001$, chemical potential $\mu=6$ and coupling constant $c=30$. The right plot shows $S-T$ curves for external magnetic field $H=0.001$, chemical potential $\mu=3$ and coupling constant $c=50$. See text.

(This figure is in colour only in the electronic version)

$$
\begin{aligned}
v_{c} & =2 n \pi\left(1-4 \pi n s_{1} * a_{1}(k)\right) \\
& =2 n \pi\left(1-\frac{2 n \pi}{3 \sqrt{3} c}-\frac{2 n \ln 3}{c}\right) .
\end{aligned}
$$

The spin and charge velocities can be derived from the relations $v_{c}=\varepsilon^{\prime}\left(k_{0}\right) / 2 \pi \rho_{c}\left(k_{0}\right)$ and $v_{s}=\phi_{1}^{(r) \prime}\left(\lambda_{0}\right) / 2 \pi \rho_{s}\left(\lambda_{0}\right)$ [50]. For three-component fermions, there are two spin velocities $v_{s 1}$ and $v_{s 2}$, where $v_{s 1}=v_{s 2}$. The central charge for the spin part is $C_{s}=2$ and that for the charge part is $C_{c}=1$. The reason for $C_{s}=2$ is because the $S U$ (3) invariant fermion model has two spin 'Fermi seas' whose dependence on $H$ are equal, i.e. we considered the case where $H_{1}=H_{2}=H$. This result shows that spin-charge separation exists for low temperatures and a small external magnetic field. This result is coincident with the results from conformal field theory $[12,11]$.

To compare the results from Sommerfeld's expansion and the polylog function, we plot graphs of specific heat $C_{V}-T$ and entropy $S_{V}-T$ versus temperature in figure 1 . Both graphs show that at low temperatures in the strong coupling regime, Sommerfeld's expansion agrees well with the polylog function. However, when the temperature increases, the deviation between the two curves become more apparent. This means that the Tomonaga-Luttinger liquid form of the free energy is only valid at very low temperatures and conformal invariance breaks down as the excitations take place at higher temperatures.

\section{Conclusion}

We have presented a systematic way to study low-temperature behavior of systems with high spin symmetry and with repulsive interactions via the Wiener-Hopf method. In particular, we have derived the universal thermodynamics of 1D strongly repulsive fermions with $S U$ (3) symmetry under a weak external magnetic field. We have applied the Wiener-Hopf method to obtain the universal low-energy physics in terms of spin-charge separation. The chemical potential (104), pressure (105) and free energy (106) of the system have been derived analytically at low temperatures. The free energy gives insight into understanding the spin-charge separation theory for the higher spin systems. Moreover, beyond the spin-charge separation regime, the thermodynamics (102) has been given in terms of the polylog function. 
This result can be used to test universal scaling behavior in the quantum critical regime. Our results open up further study of quantum criticality in 1D interacting Fermi gases with higher spin symmetry and repulsive interaction.

\section{Acknowledgments}

This work is in part supported by the NSFC, the Knowledge Innovation Project of Chinese Academy of Sciences, the National Program for Basic Research of MOST(China) and the Australian Research Council.

\section{Appendix A. Solution to equation (79)}

With the product decomposition given in (81), equation (79) can be written as

$$
\mathbf{G}_{+}^{-1}(\omega) \hat{\mathbf{S}}(\omega)=\mathbf{G}_{-}(\omega) \hat{\mathbf{f}}_{\mathbf{S}}(\omega)+\mathbf{G}_{-}(\omega) \hat{\mathbf{b}}_{\mathbf{S}}(\omega) .
$$

The function $\mathbf{G}_{+}^{-1}(\omega) \hat{\mathbf{S}}(\omega)$ is analytic on the upper-half plane by the definitions of $\mathbf{G}_{+}^{-1}(\omega)$ and $\hat{\mathbf{S}}(\omega)$. On the other hand, $\mathbf{G}_{-}(\omega) \hat{\mathbf{b}}_{\mathbf{S}}(\omega)$ is analytic on the lower-half plane. We are left with the function $\mathbf{G}_{-}(\omega) \hat{\mathbf{f}}_{\mathbf{S}}(\omega)$ which we have to decompose into the sum of a function which is analytic on the upper-half plane and another function which is analytic on the lower-half plane.

First, note that $\hat{f}_{S}^{(r)}(\omega)$ can be written as

$$
\hat{f}_{S}^{(r)}(\omega)=\frac{2 \pi P \mathrm{i}}{\sqrt{3} c}\left(\frac{1}{\omega+\mathrm{i} \epsilon}-\frac{\kappa^{(r)}}{\omega+2 \pi \mathrm{i} / 3 c}\right)
$$

where $\epsilon$ is small. The function $\mathbf{G}_{-}(\omega) \hat{\mathbf{f}}_{\mathbf{S}}(\omega)$ in matrix form is then decomposed into

$$
\begin{aligned}
\mathbf{G}_{-}(\omega) \hat{\mathbf{f}}_{\mathbf{S}}(\omega)= & \frac{2 \pi P \mathrm{i}}{\sqrt{3} c}\left[\frac{1}{\omega+\mathrm{i} \epsilon}\left(\mathbf{G}_{-}(\omega)-\mathbf{G}_{-}(-\mathrm{i} \epsilon)\right)\left(\begin{array}{l}
1 \\
1
\end{array}\right)\right. \\
& \left.-\frac{1}{\omega+2 \pi \mathrm{i} / 3 c}\left(\mathbf{G}_{-}(\omega)-\mathbf{G}_{-}(-2 \pi \mathrm{i} / 3 c)\right)\left(\begin{array}{c}
\kappa^{(1)} \\
\kappa^{(2)}
\end{array}\right)\right] \\
& +\frac{2 \pi P \mathrm{i}}{\sqrt{3} c}\left[\frac{1}{\omega+\mathrm{i} \epsilon} \mathbf{G}_{-}(-\mathrm{i} \epsilon)\left(\begin{array}{l}
1 \\
1
\end{array}\right)-\frac{1}{\omega+2 \pi \mathrm{i} / 3 c} \mathbf{G}_{-}(-2 \pi \mathrm{i} / 3 c)\left(\begin{array}{c}
\kappa^{(1)} \\
\kappa^{(2)}
\end{array}\right)\right] \\
\equiv & \boldsymbol{\Phi}^{-}(\omega)+\boldsymbol{\Phi}^{+}(\omega) .
\end{aligned}
$$

Thus, (A.1) can be rewritten as

$$
\mathbf{G}_{+}^{-1}(\omega) \hat{\mathbf{S}}(\omega)-\boldsymbol{\Phi}^{+}(\omega)=\boldsymbol{\Phi}^{-}(\omega)+\mathbf{G}_{-}(\omega) \hat{\mathbf{b}}_{\mathbf{S}}(\omega)
$$

where the left-hand side is analytic on the upper-half plane, and right-hand side is analytic on the lower-half plane. When $\omega \rightarrow \infty$, both the left-hand side and right-hand side tend to zero; thus

$$
\mathbf{G}_{+}^{-1}(\omega) \hat{\mathbf{S}}(\omega)-\boldsymbol{\Phi}^{+}(\omega)=0
$$

which gives

$$
\begin{array}{r}
\hat{\mathbf{S}}(\omega)=\mathbf{G}_{+}(\omega) \boldsymbol{\Phi}^{+}(\omega)=\frac{2 \pi P \mathrm{i}}{\sqrt{3} c}\left[\frac{1}{\omega+\mathrm{i} \epsilon} \mathbf{G}_{+}(\omega) \mathbf{G}_{-}(0)\left(\begin{array}{l}
1 \\
1
\end{array}\right)\right. \\
\left.-\frac{1}{\omega+2 \pi \mathrm{i} / 3 c} \mathbf{G}_{+}(\omega) \mathbf{G}_{-}(-2 \pi \mathrm{i} / 3 c)\left(\begin{array}{c}
\kappa^{(1)} \\
\kappa^{(2)}
\end{array}\right)\right] .
\end{array}
$$


Since $S^{(r)}(0)=0$ and $\hat{S}^{(r)}(\omega)$ is analytic on the upper-half plane, the following result

$$
\lim _{|\omega| \rightarrow \infty} \omega \hat{\mathbf{S}}(\omega)=0
$$

is given by contour integration. This boundary condition is equivalent to

$$
\mathbf{G}_{-}(-2 \pi \mathrm{i} / 3 c)\left(\begin{array}{l}
\kappa^{(1)} \\
\kappa^{(2)}
\end{array}\right)=\mathbf{G}_{-}(0)\left(\begin{array}{l}
1 \\
1
\end{array}\right) .
$$

Using this result we can show that

$$
\hat{\mathbf{S}}(\omega)=\mathrm{i} \frac{2 \pi P}{\sqrt{3} c}\left(\frac{1}{\omega+\mathrm{i} \epsilon}-\frac{1}{\omega+2 \pi \mathrm{i} / 3 c}\right) \mathbf{G}_{+}(\omega) \mathbf{G}_{-}(0)\left(\begin{array}{l}
1 \\
1
\end{array}\right) .
$$

Besides, note that

$$
\mathbf{S}^{\prime}(0)=\left.\frac{\mathrm{d}}{\mathrm{d} \lambda} \mathbf{S}(\lambda)\right|_{\lambda=0^{+}}=-\lim _{|\omega| \rightarrow \infty} \omega^{2} \hat{\mathbf{S}}(\omega)
$$

thus we find the expression of $\mathbf{S}^{\prime}(0)$

$$
\mathbf{S}^{\prime}(0)=\frac{4 \pi^{2} P}{3 \sqrt{3} c^{2}} \mathbf{G}_{-}(0)\left(\begin{array}{l}
1 \\
1
\end{array}\right)
$$

\section{Appendix B. Solution to equation (80)}

Similarly for $\hat{\mathbf{T}}(\omega)$ with the factorization (81), we find that

$$
\mathbf{G}_{+}^{-1}(\omega) \hat{\mathbf{T}}(\omega)=\mathbf{G}_{-}(\omega) \hat{\mathbf{f}}_{\mathbf{T}}(\omega)+\mathbf{G}_{-}(\omega) \hat{\mathbf{b}}_{\mathbf{T}}(\omega) .
$$

With similar arguments as before, we only need to find a decomposition of $\mathbf{G}_{-}(\omega) \hat{\mathbf{f}}_{\mathbf{T}}(\omega)$ into the sum of two functions that are analytic on the upper-half plane and lower-half plane, respectively. Consider the functions

$$
\begin{aligned}
& f_{T}^{(1)}(\lambda)=\frac{h(\lambda)}{S^{(1) \prime}(0)}+\frac{g\left(\lambda+\lambda_{0}^{(1)}-\lambda_{0}^{(2)}\right)}{S^{(2) \prime}(0)}, \\
& f_{T}^{(2)}(\lambda)=\frac{h(\lambda)}{S^{(2) \prime}(0)}+\frac{g\left(\lambda+\lambda_{0}^{(1)}-\lambda_{0}^{(2)}\right)}{S^{(1)^{\prime}}(0)} .
\end{aligned}
$$

After taking a Fourier transformation, the matrix $\hat{\mathbf{f}}_{\mathbf{T}}(\omega)$ can be expressed in terms of the kernel $\hat{\mathbf{K}}(\omega)$ as

$$
\hat{\mathbf{f}}_{\mathbf{T}}(\omega)=\hat{\mathbf{K}}(\omega) \mathbf{V}=\left(\mathbf{I}-\mathbf{G}_{-}^{-1}(\omega) \mathbf{G}_{+}^{-1}(\omega)\right) \mathbf{V}
$$

where

$$
\mathbf{V}=\left(\begin{array}{l}
1 / S^{(1) \prime}(0) \\
1 / S^{(2) \prime}(0)
\end{array}\right)
$$

Multiplying $\mathbf{G}_{-}(\omega)$ on both sides of this equation, we have

$$
\mathbf{G}_{-}(\omega) \hat{\mathbf{f}}_{\mathbf{T}}(\omega)=\left(\mathbf{G}_{-}(\omega)-\mathbf{G}_{+}^{-1}(\omega)\right) \mathbf{V}
$$

Substituting this relation back into equation (B.1) gives

$$
\mathbf{G}_{+}^{-1}(\omega) \hat{\mathbf{T}}(\omega)+\mathbf{G}_{+}^{-1}(\omega) \mathbf{V}=\mathbf{G}_{-}(\omega) \mathbf{V}+\mathbf{G}_{-}(\omega) \hat{\mathbf{b}}_{\mathbf{T}}(\omega)
$$

Based on analyticity arguments, matrix elements on both sides of the equation are equal to a function that we shall denote in matrix form by $\mathbf{Q}(\omega)$, i.e.

$$
\mathbf{G}_{+}^{-1}(\omega) \hat{\mathbf{T}}(\omega)+\mathbf{G}_{+}^{-1}(\omega) \mathbf{V}=\mathbf{Q}(\omega)
$$

Similarly to $\hat{\mathbf{S}}(\omega)$, the function $\hat{\mathbf{T}}(\omega)$ vanishes when $\omega \rightarrow \infty$. Because $\mathbf{Q}(\omega)$ is an entire function that is bounded, it is equal to a constant by Louville's theorem. This constant can be determined by taking the limit $\omega \rightarrow \infty$ which gives $\mathbf{Q}=\mathbf{V}$. Hence we finally obtain an equation for $\hat{\mathbf{T}}(\omega)$ expressed in terms of functions whose values are known, i.e.

$$
\hat{\mathbf{T}}(\omega)=\left(\mathbf{G}_{+}(\omega)-\mathbf{I}\right) \mathbf{V}
$$




\section{References}

[1] Paredes B, Widera A, Murg V, Mandel O, Fölling S, Cirac I, Shlyapnikov G V, Hänsch T W and Bloch I 2004 Nature 429277

[2] Kinoshita T, Wenger T and Weiss D S 2004 Science 3051125

[3] Moritz H, Stöferle T, Günter K, Köhl M and Esslinger T 2005 Phys. Rev. Lett. 94210401

[4] Haller E, Gustavsson M, Mark M J, Danzl J G, Hart R, Pupillo G and Nägerl H-C 2009 Science 3251224

[5] Liao Y, Rittner A S C, Paprotta T, Li W, Partridge G B, Hulet R G, Baur S K and Mueller E J 2010 Nature 467567

[6] Takahashi M 1999 Thermodynamics of One-Dimensional Solvable Models (Cambridge: Cambridge University Press)

[7] Giamarchi T 2004 Quantum Physics in One Dimension (Oxford: Oxford University Press)

[8] Recati A, Fedichev P O, Zwerger W and Zoller P 2003 Phys. Rev. Lett. 90020401

[9] Fuchs J N, Gangardt D M, Keilmann T and Shlyapnikov G V 2005 Phys. Rev. Lett. 95150402

[10] Kollath C, Schollwöck U and Zwerger W 2005 Phys. Rev. Lett. 95176401

[11] Blöte H W J, Cardy J L and Nightingale M P 1986 Phys. Rev. Lett. 56742

[12] Affleck I 1986 Phys. Rev. Lett. 56746

[13] Guan X-W, Batchelor M T, Lee C and Bortz M 2007 Phys. Rev. B 76085120

[14] Zhao E, Guan X-W, Vincent Liu W, Batchelor M T and Oshikawa M 2009 Phys. Rev. Lett. 103140404

[15] Lee J Y, Guan X W, Sakai K and Batchelor M T 2011 arXiv:1104.2352v1

[16] Fulde P and Ferrell R A 1964 Phys. Rev. 135 A550

Larkin A I and Ovchinnikov Y N 1965 Sov. Phys._JETP 20762

[17] Orso G 2007 Phys. Rev. Lett. 98070402

[18] Hu H, Liu X-J and Drummond P D 2007 Phys. Rev. Lett. 98070403

[19] Iida T and Wadati M 2008 J. Phys. Soc. Japan 77024006

[20] Casula M, Ceperley D M and Mueller E J 2008 Phys. Rev. A 78033607

[21] Tezuka M and Ueda M 2008 Phys. Rev. Lett. 100110403

[22] Feiguin A E and Heidrich-Meisner F 2008 Phys. Rev. B 76220508

[23] Gao X and Asgari R 2008 Phys. Rev. A 77033604

[24] Rizzi M, Polini M, Cazalilla M A, Bakhtiari M R, Tosi M P and Fazio R 2008 Phys. Rev. B 77245105

[25] Batrouni G G, Huntley M H, Rousseau V G and Scalettar R T 2008 Phys. Rev. Lett. 100116405

[26] Kinunnen J, Jensen L M and Törmä P 2006 Phys. Rev. Lett. 96110403

[27] Zhao E and Liu W V 2008 Phys. Rev. A 78063605

[28] Gorshkov A V, Hermele M, Gurarie V, Xu C, Julienne P S, Ye J, Zoller P, Demler E, Lukin M D and Rey A M 2010 Nature Phys. 6289

Cazalilla M A, Ho A F and Ueda M 2009 New J. Phys. 11103033

[29] Taie S, Takasu Y, Sugawa S, Yamazaki R, Tsujimoto T, Murakami R and Takahashi Y 2010 Phys. Rev. Lett. 105190401

[30] Ho T-L 1998 Phys. Rev. Lett. 81742

[31] Ho T-L and Yip S 1999 Phys. Rev. Lett. 82247

[32] Guan X-W, Batchelor M T, Lee C and Zhou H-Q 2008 Phys. Rev. Lett. 100200401

[33] Guan X-W, Lee J-Y, Batchelor M T, Yin X G and Chen S 2010 Phys. Rev. A 82 021606(R)

[34] He P, Yin X-G, Guan X-W, Batchelor M T and Wang Y 2010 Phys. Rev. A 82053633

[35] Lieb E H and Liniger W 1963 Phys. Rev. 1301605

[36] Yang C N 1967 Phys. Rev. Lett. 191312

[37] Gaudin M 1967 Phys. Lett. A 2455

[38] Sutherland B 1968 Phys. Rev. Lett. 2098

[39] Yang C N 1970 Lectures given at the Karpacz Winter School of Physics see Yang C N 1983 Selected Papers 1945-1980 (San Francisco: Freeman) p 430

[40] Takahashi M 1970 Prog. Theor. Phys. 44899

[41] Mezincescu L, Nepomechie R I, Townsend P K and Tsvelik A M 1993 Nucl. Phys. B 406681

[42] Mezincescu L and Nepomechie R I 1992 arXiv:hep-th/9212124v1

[43] Controzzi D and Tsvelik A M 2006 Phys. Rev. Lett. 96097205

[44] Wu C, Hu J-P and Zhang S-C 2003 Phys. Rev. Lett. 91186402 Wu C 2005 Phys. Rev. Lett. 95266404

[45] Jiang Y, Cao J and Wang Y 2009 Europhys. Lett. 8710006

[46] Friedan D, Qiu Z and Shenker S 1984 Phys. Rev. Lett. 521575 Affleck I 1985 Phys. Rev. Lett. 551355 
Belavin A A, Polyakov A M and Zamolodchikov A B 1984 Nucl. Phys. B 241333

Sen D 1991 Phys. Rev. B 442645

[47] Kawakami N and Yang S-K 1991 J. Phys.: Condens. Matter. 35983

[48] Frahm H and Korepin V E 1990 Phys. Rev. B 4210553

Frahm H and Korepin V E 1991 Phys. Rev. B 435653

[49] Koponen T K, Paananen T, Martikainen J-P and Törmä P 2007 Phys. Rev. Lett. 99120403

[50] Gohberg I C and Krein M G 1960 American Mathematical Society Translations: Series 2 vol 14 (Washington, DC: American Mathematical Society) p 217

Krein M G 1962 American Mathematical Society Translations: Series 2 vol 22 (Washington, DC: American Mathematical Society) p 163 